\title{
Comments on: The role of vitamin $D$ in the prevention of coronavirus disease 2019 infection and mortality
}

\author{
Antonello Maruotti ${ }^{1,2}$ - Filippo Belloc ${ }^{3} \cdot$ Antonio Nicita $^{4,5}$
}

Received: 16 May 2020 / Accepted: 30 May 2020 / Published online: 11 July 2020

(c) Springer Nature Switzerland AG 2020

The COVID-19 pandemic has been coupled worldwide with an explosion of information about the virus. In its present form, COVID-19 seems to have two very challenging characteristics [1]: it is highly infectious and, despite having a benign course in the vast majority of patients, it requires hospital admission and even intensive care for a far from negligible proportion of infected. This has generated a lot of publications at a rapid pace, addressing and investigating different aspects of the epidemic [see, e.g., 2- 7] and, sadly, also an increase in misinformation, that is of course not all malevolent, although its impact can be devastating [8,9].

Across all the huge literature on the possible determinants leading to a reduction of the infection, fatality, and mortality rates, our attention has been attracted by the work of Ilie et al. on the role of vitamin D in the prevention of COVID19 infection and mortality [10]. Vitamin D is known to play an important role in bone metabolism through regulation of calcium and phosphate homeostasis, and may also play an important role in immune system regulation. Vitamin D is produced by the body during exposure to sunlight, but is also found in oily fish, eggs, and fortified food products. In addition to causing rickets, vitamin $\mathrm{D}$ deficiency has been linked to respiratory infections $[10,11]$. Some studies have suggested that vitamin D supplementation can decrease the

Commissioner Autorità per Garanzie nelle Comunicazioni.

Antonello Maruotti

a.maruotti@lumsa.it; antonello.maruotti@uib.no

1 Dipartimento GEPLI, Libera Università Maria Ss Assunta, Rome, Italy

2 Department of Mathematics, University of Bergen, Bergen, Norway

3 Dipartimento di Economia Politica e Statistica, Università di Siena, Siena, Italy

4 Dipartimento di Giurisprudenza, Libera Università Maria Ss Assunta, Palermo, Italy

5 Autorità per le Garanzie nelle Comunicazioni, Rome, Italy frequency and severity of respiratory infections; however, further research is needed before specific recommendations can be made [12-14]. According to these general premises, the work of Ilie et al. may play a fundamental role in limiting the spread of COVID-19 and reducing the number of deaths [15]. The authors clearly state that the crude association observed in the present study may be explained by the role of vitamin $D$ in the prevention of COVID-19 infection or more probably by a potential protection of vitamin $D$ from the more negative consequences of the infection. This is a rather neat and important statement, that may strongly contribute to handle the pandemic outcomes properly.

Unfortunately, however, we cast some doubts on the statistical methods employed on which this statement is based. The fundamental statement above is based on a correlation test, though it implicitly implies a cause-effect relationship. However, correlation does not imply causation, that can be investigated, e.g., in a regression setting. We reproduced the analysis published in the work of Ilie et al. [15]. We noticed the following:

- The correlation between levels of mean vitamin D and the number of deaths caused by COVID-19/1 M population in each country is correctly reported $\rho=-0.4378$. Nevertheless, the correct $\mathrm{p}$ value is not the one reported in the paper. The correct $\mathrm{p}$ value is 0.05353 , above the nominal significance $5 \%$ level.

- The correlation between levels of mean vitamin D and the number of cases of COVID-19/1 M population in each country is correctly reported $\rho=-0.4435$. Nevertheless, the reported $\mathrm{p}$ value is slightly above the one reported in the paper. The correct $\mathrm{p}$ value is 0.05014 , slightly above the nominal significance $5 \%$ level.

The significant cut point $p$ value $<0.05$ is not achieved.

As a general matter, correlations must be interpreted with a lot of care $[16,17]$. Pearson himself $[18,19]$ suggested to be very wary of correlating ratios and, if forced to do so, to 
adopt as the point of no connection not 0 , but some value as 0.4. Furthermore, to properly apply the hypothesis testing on the correlation coefficient, some assumptions must be fulfilled. Just to mention violations which potentially affect the results:

- the data do not follow a Gaussian distribution;

- there is heteroscedasticity, and the variance is smaller for a particular range of values and larger for another range of values;

- outliers are present, they can significantly skew the correlation coefficient and make it inaccurate.

The data analysis provided by Ilie et al. [15] has a few more statistical-related weaknesses, some correctly acknowledged by the authors. We mention just a few with the aim of providing a basis for a revision of the data analysis on a more solid statistical ground. First, as discussed also by the authors, data are heterogeneous, i.e., the sample is not drawn from a single population but rather from a mixture. This could be due to the country-specific data collection processes, e.g., on COVID-19 deaths: for example, in Italy, recorded deaths are with COVID-19, while in Germany, recorded deaths are by COVID-19. Moreover, as the epidemic started at different dates in different countries, the time at which the data are collected may also play a role, increasing unobserved heterogeneity. However, if heterogeneity is not taken into account, the obtained results may be unreliable, whatever statistical approach is considered. Second, if a linear relationship/ model is considered, fitted values may likely be negative for the data at hand (for Slovakia, the fitted linear predictor is -16.04316 deaths/1M population, i.e., unreliable), and this is not possible as the data are defined on the positives only. This issue can be easily solved by considering a generalized linear model for counts, with an offset. At last, as a further point for discussion, we would remark that there is a large repertoire of methods based on different paradigms of inference that provide ample options for supplementing and enhancing simple hypothesis testing [20]. Thus, even if Ilie et al. pose the basis for a novel and potentially very useful strand of research [15], there is still a lot to do on the statistical ground to be confident on any significant relationship between vitamin D and COVID-19 epidemic.

The main conclusion that we found significant crude relationships between vitamin D levels and the number COVID19 cases and especially the mortality caused by this infection is currently not supported by the data analysis. Of course, the relationship between vitamin D and COVID-19 deserves dedicated studies, as correctly discussed by Ilie et al. [15], as it may reveal interesting insights on the COVID-19 outbreak. This is a very interesting field of research with no doubts, and further studies should be planned. Currently, however, there is no evidence of any effects of vitamin $\mathrm{D}$ in reducing the impact of COVID-19 on the number of cases neither on deaths.

\section{Compliance with ethical standards}

Funding No source of funding was used.

Conflict of interest We declare that we have no conflict of interest.

Ethical statement This study is compliant with the ethical standards.

Informed consent Considering the design of the study, no informed consent was necessary.

\section{References}

1. Peeri NC, Shrestha N, Rahman S et al (2020) The SARS, MERS and novel coronavirus (COVID-19) epidemics, the newest and biggest global health threats: what lessons have we learned? Int J Epidemiol. https://doi.org/10.1093/ije/dyaa033

2. Böhning D, Rocchetti I, Maruotti A, and Holling H (2020) Estimating the undetected infections in the Covid-19 outbreak by harnessing capture-recapture methods. Int J Infect Dis. https:// doi.org/10.1016/j.ijid.2020.06.009

3. Giordano G, Blanchini F, Bruno R, Colaneri P, Di Filippo A, Di Matteo A, Colaneri M (2020) Modelling the COVID-19 epidemic and implementation of population-wide interventions in Italy. Nat Med 26:855-860. https://doi.org/10.1038/s41591-020-0883-7

4. Giorgi PD, Schiró GR, Capitani D, D'Aliberti G, Gallazzi E (2020) Vertebral compression fractures in multiple myeloma: redefining the priorities during the COVID-19 pandemic. Aging Clin Exp Res. https://doi.org/10.1007/s40520-020-01590-4

5. Nanda A, Vura NVRK, Gravenstein S (2020) COVID-19 in older adults. Aging Clin Exp Res. https://doi.org/10.1007/s40520-02001581-5

6. Onder G, Rezza G, Brusaferro S (2020) Case-fatality rate and characteristics of patients dying in relation to COVID-19 in Italy. JAMA 323:1775-1776. https://doi.org/10.1001/jama.2020.4683

7. Tuite AR, Ng V, Rees E et al (2020) Estimation of COVID19 outbreak size in Italy. Lancet Infect Dis 20:537. https://doi. org/10.1016/S1473-3099(20)30227-9

8. Garrett L (2020) (2020) COVID-19: the medium is the message. Lancet 395:942-43

9. Limaye RJ, Sauer M, Ali J et al (2020) Building trust while influencing online COVID-19 content in the social media world. Lancet Digit Health 2:e277-e278

10. Cannell JJ, Vieth R, Umhau JC et al (2006) Epidemic influenza and vitamin D. Epidemiol Infect 356:1129-1140

11. Gruber-Bzura BM (2018) Vitamin D and influenza-prevention or therapy? Int J Mol Sci 19:2419

12. Bergman P, Lindh AU, Björkhem-Bergman L, et al (2013) Vitamin D and respiratory tract infections: a systematic review and meta-analysis of randomized controlled trials. PLoS-One 8:e65835

13. Charan J, Goyal JP, Saxena D, et al (2012) Vitamin D for prevention of respiratory tract infections: a systematic review and metaanalysis. J Pharmacol Pharmacother 3:300-303

14. Martineau Adrian R, Jolliffe David A, Richard HL et al (2017) Vitamin D supplementation to prevent acute respiratory tract 
infections: systematic review and meta-analysis of individual participant data. BMJ 356:i6583

15. Ilie PC, Stefanescu S, Smith L (2020) The role of vitamin D in the prevention of coronavirus disease 2019 infection and mortality. Aging Clin Exp Res. https://doi.org/10.1007/s40520-020-01570 $-8$

16. Aldrich J (1995) Correlations genuine and spurious in Pearson and Yule. Stat Sci 10:364-376

17. Vigen T (2015) Spurious correlations. Hachette Books ISBN: 9780316339438
18. Pearson K (1897) On a form of spurious correlation which may arise when indices are used in the measurement of organs. Proc R Soc Lond 60:489-498

19. Pearson K (1902) On the mathematical theory of errors of judgment, with special reference to the personal equation. Philos Trans R Soc Ser A 198:235-299

20. Wellek S (2017) A critical evaluation of the current " $p$-value" controversy. Biom J 59:854-872 